\title{
Calidad de vida, emociones negativas, autoeficacia y calidad del sueño en pacientes con dolor crónico: efectos de un programa de intervención psicológica
}

\section{Quality of Life, Negative Emotions, Self-Efficacy and Quality of Sleep in Patients with Chronic Pain: Effects of a Program of Psychological Intervention}

\author{
Carlos Javier van-der Hofstadt \\ ROMÁN \\ Universidad Miguel Hernández, España \\ Instituto de Investigación Sanitaria y Biomédica \\ de Alicante (Isabial-Fisabio), España \\ Cesar Leal-Costa ${ }^{\mathrm{a}}$ \\ Universidad Católica de Murcia, España \\ ORCID: orcid.org/0000-0002-7711-3877 \\ Miguel Raúl Alonso-Gascon \\ Universidad Miguel Hernández, España \\ Jesús RodríGuez-Marín \\ Universidad Miguel Hernández, España \\ Instituto de Investigación Sanitaria y Biomédica \\ de Alicante (Isabial-Fisabio), España
}

Recepción: 17 Diciembre 2016 | Aprobación: 04 Febrero 2017

\footnotetext{
a Autor de correspondencia. Correo electrónico: cjvander@umh.es

Para citar este artículo: Van-der Hofstadt Román, C. J., Leal-Costa, C., Alonso-Gascón, M. R., \& Rodríguez-Marín, J. (2017). Calidad de vida, emociones negativas, autoeficacia y calidad del sueño en pacientes con dolor crónico: efectos de un programa de intervención psicológica. Universitas Psychologica, 16(3), 1-9.

https://doi.org/10.11144/Javeriana.upsy16-3.cven
}

\section{RESUMEN}

El dolor crónico es un problema de salud que afecta a entre el 10 y el $30 \%$ de la población. El objetivo del presente estudio es evaluar la eficacia de un programa de intervención grupal en dolor crónico. Se realizó un estudio cuasi experimental. La muestra estuvo compuesta por 105 participantes de la Unidad de Dolor del Hospital General Universitario de Alicante. Se administró una batería de cuestionarios pre y post a la aplicación del programa. En el estudio se midió el nivel de dolor, calidad de vida, ansiedad, depresión, atributos del sueño y autoeficacia. Se obtuvieron mejoras significativas en los niveles de ansiedad, depresión calidad de vida física, autoeficacia, en todas sus dimensiones (control de síntomas, funcionamiento físico y control del dolor) y aspectos de los atributos del sueño, como cantidad y alteraciones del sueño. El programa protocolizado y realizado por profesionales entrenados es efectivo para disminuir la percepción del dolor, aumentar la calidad de vida en su componente físico, mejorar la autoeficacia y los atributos del sueño en pacientes con dolor crónico.

Palabras clave

dolor crónico; ansiedad; depresión; autoeficacia; calidad de vida; sueño

\begin{abstract}
Chronic pain is a health problem that affects between 10 and $30 \%$ of the population. The goal of this study is to evaluate the effectiveness of a group intervention program for chronic pain. A quasi-experimental study was carried out. The sample consists of 105 participants of the Pain Unit of the Hospital General Universitario de Alicante. A battery
\end{abstract}


of questionnaires pre and post was administered to the application of the program. The study measured the level of pain, quality of life, anxiety, depression, sleep attributes and self-efficacy. Significant improvements in levels of anxiety, depression, physical quality of life, self-efficacy in all dimensions (symptom control, physical functioning and pain control) and aspects of sleep attributes such as sleep disturbances were obtained. The program protocolized and carried out by trained professionals is effective to decrease the perception of pain, to increase the quality of life in its physical component, to improve the self-efficacy and the attributes of the dream with chronic pain patients.

Keywords

Chronic pain; anxiety; depression; self-efficacy; quality of life; sleep

\section{Introducción}

El dolor crónico es una de las principales causas de consulta que reciben los profesionales sanitarios. Los datos sobre su prevalencia son muy heterogéneos y suelen oscilar entre el 10 y el $30 \%$ de la población adulta (Breivik, Collett, Ventafridda, Cohen, \& Gallacher, 2006), no existiendo diferencias relevantes en función del sexo. La primera revisión epidemiológica de dolor crónico no oncológico en España refleja que más de seis millones de españoles sufren dolor, lo que representa el $17.25 \%$ de la población adulta, aunque existen diferencias en función de la localización geográfica, entre el $18.79 \%$ y el $14.8 \%$, siendo preponderantes dos tipos: el dolor de espalda (60.53\%) y el dolor osteoarticular (40.21\%) (Soares-Weiser et al., 2010).

En los pacientes con dolor crónico, los aspectos emocionales, conductuales y cognitivos interrelacionados determinan en gran medida la experiencia de dolor (Bettina et al., 2008). Esta asociación se refleja ampliamente en la literatura en relación con la depresión, la ansiedad, la calidad de vida, la autoeficacia y la alteración del sueño (Boschen et al., 2016; McCracken, 2012). Así, por ejemplo, en el $42.24 \%$ de los casos aparecen alteraciones del sueño, ansiedad en el $40.62 \%$ y estados depresivos en el $24.43 \%$ de los casos (Soares-Weiser et al., 2010).

En los estándares y recomendaciones del Ministerio de Sanidad, Servicios Sociales e Igualdad de España sobre el tema, se plantea claramente que las unidades multidisciplinares para el tratamiento del dolor: "Debe[n] contar con un psicólogo o psiquiatra integrado en la plantilla de la unidad o con la disponibilidad del mismo para valorar y tratar los aspectos psíquicos y psicosociales del paciente con dolor crónico" (Palanca-Sánchez et al., 2011, p. 63), con una formación especializada en dolor. Asî mismo, el Ministerio recoge las competencias, conocimientos y habilidades que este debe poseer, e indica claramente entre sus funciones la "administración de terapia o tratamiento cognitivo-conductual, y de otras terapias que han demostrado su eficacia en el tratamiento del dolor como la hipnosis y biofeedback" (PalancaSánchez et al., 2011, p. 103). De igual forma, en una reciente revisión sobre las recomendaciones basadas en la evidencia de diferentes guías de práctica clínica sobre el abordaje del dolor lumbar crónico, la terapia cognitivo conductual, individual o grupal presenta recomendaciones a favor en todas las guías revisadas, menos en una, en la que se aconseja no utilizarla como único tratamiento, sino en combinación con otras terapias (Sauer, Burris, \& Carlson, 2010).

Estas recomendaciones, referidas al tipo de tratamiento psicológico a aplicar, coinciden con las que desde hace tiempo se vienen realizando desde la literatura científica, siendo el tratamiento cognitivo conductual, con sus diferentes desarrollos, el considerado como tratamiento de elección (Boschen et al., 2016; Eccleston, Hearn, \& Williams, 2016; Inoue et al., 2014; McCracken, 2012; Moix \& Casado, 2011; Moix \& Kovacs, 2009).

Sin embargo, la presencia de psicólogos especializados en tema en las unidades de tratamiento de dolor es excepcional. Lo más habitual es una colaboración puntual por parte de estos profesionales.

Esta situación nos lleva a diseñar y poner a disposición de profesionales no especializados en la temática un programa de intervención grupal multicomponente para pacientes con dolor crónico moderado no oncológico (RodríguezMarín, van-der Hofstadt, \& Couceiro, 2013), totalmente protocolizado y con todos los elementos necesarios para poder ser aplicado con un mínimo entrenamiento previo. Este programa 
integra diferentes elementos de los tratamientos cognitivos conductuales.

El objetivo general de este trabajo es evaluar la efectividad de dicho programa de intervención por profesionales no especializados en dolor crónico, que incluye procedimientos de relajación y técnicas cognitivo-conductuales, mediante la evaluación de los niveles de dolor, ansiedad, depresión, autoeficacia, calidad de vida y atributos del sueño.

\section{Material y método}

Se realizó un estudio cuasi experimental de medidas repetidas pre y postest. El diseño implica una comparación intragrupo con mediciones antes y después del programa de intervención.

\section{Participantes}

La muestra fue seleccionada mediante muestreo no aleatorio incidental de pacientes de la Unidad de Dolor del Hospital General de Alicante. Los criterios de inclusión en el estudio fueron: 1) los sujetos deben ser mayores de 20 años en el momento de la inclusión del estudio; 2) dolor crónico de intensidad moderada de más de seis meses de evolución no oncológico; 3) que el dolor interfiera al menos en un área del funcionamiento cotidiano; 4) aceptación libre de participar en el estudio, con consentimiento informado por escrito del paciente, y 5) autonomía suficiente para rellenar las pruebas autoadministradas. Los criterios de exclusión fueron: 1) sujetos con capacidades disminuidas para dar su consentimiento informado; 2) sujetos sobre los que se prevea que vayan a ser intervenidos durante la duración del estudio; 3 ) sujetos que presenten comorbilidad clínica grave, y 4) sujetos que presenten patología psiquiátrica grave o en fase aguda.

\section{Instrumentos}

Para la recolección de los datos, se utilizó un cuestionario autoadministrado que incluía los siguientes datos:

Características sociodemográficas (edad, sexo, estado civil y ocupación); diseñado ad hoc para el desarrollo del estudio.

Escala Visual Analógica (EVA). Se entendió este instrumento como un indicador cuantitativo del dolor, cuya puntuación oscila de cero (no dolor) a diez (el peor dolor imaginable). Se considera como dolor leve una EVA menor que cuatro, dolor moderado entre cuatro y seis, y dolor severo mayor que seis.

Escala Hospitalaria de Ansiedad y Depresión (HAD, por sus siglas en inglés) (Zigmond \& Snaith, 1983). Validada en población española con dolor crónico (Cabrera, Martín-Aragón, Terol, Núñez, \& Pastor, 2015). Instrumento autoaplicado de catorce ítems. La intensidad o frecuencia del síntoma se evalúa en una escala de Likert de cuatro puntos (rango 0-3). El rango de puntuación para las variables ansiedad y depresión es de 0-21, y de 0-42 para puntuación global. Para las subescalas de ansiedad y depresión se considera que las puntuaciones obtenidas entre 0.7 indican la ausencia de sintomatología, entre 8-10, caso dudoso y mayores a 11, problema clínico.

Cuestionario Short Form (SF) 12, sobre el Estado de Salud (Ware, Kosinski, Turner-Bowker, \& Gandek, 2002). Se utilizó la adaptación española (Alonso et al., 1998). Consta de doce ítems provenientes de la escala SF-36. La escala de respuesta es tipo Likert que evalúa intensidad o frecuencia. El número de opciones de respuesta varía entre tres y seis, según el ítem. La versión 2 permite obtener una puntuación para calidad de vida física y otra para calidad de vida mental, además de las ocho dimensiones del SF-36. Para cada una de estas, los ítems son codificados, agregados y transformados en una escala que tiene un rango de cero (peor estado de salud para esa dimensión), hasta cien (el mejor estado de salud). 
Cuestionario de Sueño Medical Outcomes Study Sleep Scale (MOS) (Hays, Martin, Sesti, $\&$ Spritzer, 2005). Se trata de un instrumento de doce ítems que explora el impacto o interferencia ocasionado por la enfermedad, un tratamiento o, en general, cualquier estímulo externo sobre los atributos de la arquitectura del sueño (idoneidad, cantidad, despertares bruscos, ronquidos, alteraciones de sueño y somnolencia). Además, mediante la suma de nueve o seis de los 12 ítems del instrumento, evalúa el índice global de interferencia del sueño que oscila entre 0 (ninguna interferencia o impacto) y 100 (máxima interferencia posible).

Escala de Autoeficacia en Dolor Crónico (Martín-Aragón et al., 1998). Este cuestionario consta de un total de 19 ítems con una escala de respuesta tipo Likert y un rango de respuesta entre 0 y 10 , donde 0 equivale a sentirse totalmente incapaz, 5 a moderadamente capaz y 10 a totalmente capaz. Se pueden obtener puntuaciones por subescalas o una puntuación general. El resultado indica que, a mayor puntuación, mayor grado de autoeficacia.

\section{Procedimiento}

Se aplicó el programa de intervención grupal multicomponente para pacientes con dolor crónico moderado, con 8 sesiones de una duración aproximada de 120 minutos y periodicidad semanal, con contenidos protocolizados (Rodríguez-Marín et al., 2013) (Tabla 1). Este programa se aplicó a un total de 17 grupos de 8 personas, entre marzo de 2011 y febrero de 2015, por parte de diversos terapeutas, profesionales en formación de posgrado, con un mínimo entrenamiento previo.

\section{TABLA 1}

Descripción del contenido del programa de intervención

\begin{tabular}{l}
\hline Sesión 1 \\
Información y presentación del programa. \\
Entrega de documentos. \\
Presentación de los asistentes al grupo. \\
Entrega de pruebas de evaluación. \\
Introducción de la Fase I de la relajación muscular progresiva de Jacobson (práctica en casa \\
dos semanas). \\
Explicación de factores psicológicos en la experiencia del dolor. \\
Discusión y tiempo para aclaración de dudas. \\
\hline Sesión 2 \\
\hline Revisión de la práctica de la Fase I de Jacobson (continuar su práctica en casa). \\
Realización de ejercicios de respiración abdominal. \\
Entrega y explicación de la hoja de autorregistro de actividades y su relación con el dolor \\
(realización en casa del autorregistro). \\
Explicación del planteamiento progresivo de objetivos (puesta en práctica en casa de las \\
metas elegidas). \\
Discusión y tiempo para aclaración de dudas. \\
\hline Sesión 3 \\
\hline Introducción Fase II de la relajación de Jacobson (práctica en casa durante dos semanas) \\
Revisión del cumplimiento de las metas pequeñas propuestas en la sesión anterior (puesta en \\
práctica en casa de las metas que correspondan). \\
Revisión del autorregistro de actividades y su relación con el dolor (realización en casa de un \\
nuevo autorregistro). \\
Explicación de la sahud comportamental ¿por qué llevar una vida ordenada? \\
Discusión y tiempo para aclarar dudas. \\
\hline Sesión 4 \\
\hline Revisión de la práctica de la Fase II de Jacobson (continuar su práctica esta semana). \\
Revisión del cumplimiento de las metas pequeĩas (realización en casa de las \\
correspondientes metas). \\
Revisión del autorregistro de actividades. \\
Entrega del autorregistro de pensamientos (realización en casa de este nuevo autorregistro). \\
Realización de ejercicios de respiración abdominal y prueba de imaginación guiada. \\
Discusión y aclaración de dudas. \\
\hline
\end{tabular}

Fuente: Rodríguez Marín et al. 2013.

TABLA 1 (Cont.)

Descripción del contenido del programa de intervención

\begin{tabular}{l} 
Sesión 5 \\
\hline Introducción Fase III de la relajación de Jacobson (puesta en práctica durante dos \\
semanas). \\
Revisar el cumplimiento de las metas pequeñas. \\
Revisión del autorregistro de pensamientos. \\
Entrega del autorregistro de pensamientos y técnicas de distracción (a realizar en casa \\
durante esta semana). \\
Exposición sobre el cambio de pensamientos y técnicas de distracción. \\
Discusión y aclaración de dudas. \\
\hline Sesión 6 \\
\hline Revisión de la Fase III de Jacobson (continuar esta semana su aplicación). \\
Revisión del autorregistro de pensamientos y las técnicas de distracción usadas (entrega del \\
mismo autorregistro para realizar durante la semana). \\
Exposición sobre técnicas de aceptación. \\
Relajación por visualización guiada. \\
Discusión y aclaración de dudas. \\
\hline Sesión 7 \\
\hline Introducción de la fase rápida de la relajación de Jacobson (aplicar durante esta semana). \\
Revisión del autorregistro de pensamientos y las técnicas de distracción usadas (entrega del \\
mismo autorregistro para realizar durante la semana). \\
Introducción breve sobre la autocrítica y ejercicio de refuerzo. \\
Discusión y aclaración de dudas. \\
\hline Sesión 8 \\
\hline Pruebas de evahuación. \\
Prevención de recaidas y refuerzo de la continuidad de la práctica de los ejercicios. \\
Valoración del programa. \\
\hline
\end{tabular}

Fuente: Rodríguez Marín et al. 2013. 
A lo largo del programa se realizó psicoeducación, facilitando información sobre el síndrome de dolor, trabajándose la regulación emocional, los procesos cognitivos que dificultan el afrontamiento y la reactividad fisiológica, ayudando a los participantes a afrontar el dolor de un modo más adaptativo y abordando el dolor en las tres dimensiones que lo componen (cognitiva, emocional y conductual) (Sauer et al., 2010). Además, cada paciente continuó su tratamiento médico, de forma independiente a su participación en el programa.

El estudio fue aprobado por el Comité de Ética de Investigación Clínica del Hospital General Universitario de Alicante. Se siguieron las normas de protección de datos de carácter personal que se establecen para los datos de salud (Ley Orgánica 15/1999, de 13 de diciembre), y recomendaciones de ámbito internacional (garantía de participación voluntaria e informada, confidencialidad de los datos, riesgos para los participantes en el estudio e información a los participantes) (Asociación Médica Mundial [AMM], 2013).

Análisis de datos

Para procesar la información se elaboró una base de datos con el programa informático SPSS ${ }^{\odot}$ v21. Se calcularon los estadísticos descriptivos (media y desviación típica) de las variables cuantitativas, así como frecuencias y porcentajes de las variables categóricas. Por otro lado, para la evaluación del programa de intervención se realizó la prueba t-Student para muestras relacionadas entre las puntuaciones de los instrumentos de medición del pre y post. Antes de realizar las pruebas se comprobó el supuesto de normalidad de las variables. Para verificar que se cumple esta condición, se aplicó a todas ellas el test de Kolmogorov-Smirnov, que mide la concordancia entre la distribución seguida por la muestra y la distribución normal. Además, se calculó el tamaño del efecto a través de la d de Cohen para valorar la magnitud del efecto de la intervención, tomándose los valores propuestos por Cohen (1988), donde 0.2 indica un tamaño del efecto pequeño, 0.5 mediano y 0.8 grande.

\section{Resultados}

La muestra final estuvo compuesta por 136 participantes, de los cuales acabaron el programa 105 , lo que supone una tasa de respuesta del $77.21 \%$. Así, de los 105 participantes, 34 (32.4 $\%)$ fueron hombres y $71(67.6 \%)$ mujeres. La media de edad fue de $51.8(\mathrm{DT}=11.06)$. En cuanto a las características sociodemográficas, observamos que hay una mayor proporción de muestra cuya situación laboral es la invalidez, su estado civil es casado y su nivel de estudios es primarios (Tabla 2).

\section{TABLA 2}

Frecuencia y porcentaje de las características sociodemográficas de la muestra

\begin{tabular}{|c|c|}
\hline Variables & n (\%) \\
\hline \multicolumn{2}{|l|}{ Sexo: } \\
\hline Hombre & $34(32.4)$ \\
\hline Mujer & $71(67.6)$ \\
\hline \multicolumn{2}{|l|}{ Estado civil: } \\
\hline Soltero/a & $13(12.4)$ \\
\hline Casado/a & $75(71.5)$ \\
\hline Divorciado/a & $10(9.5)$ \\
\hline Viudo/a & $7(6.7)$ \\
\hline \multicolumn{2}{|l|}{ Situación laboral: } \\
\hline Activo & $11(10.5)$ \\
\hline Baja & $13(12.4)$ \\
\hline Paro & $13(12.4)$ \\
\hline Invalidez & $36(34.3)$ \\
\hline Jubilado & $23(21.9)$ \\
\hline Ama de casa & $9(8.6)$ \\
\hline \multicolumn{2}{|l|}{ Estudios: } \\
\hline Sin estudios & $12(11.4)$ \\
\hline Primarios & 46 (43.9) \\
\hline Secundarios & $27(25.7)$ \\
\hline Superiores & 20 (19) \\
\hline
\end{tabular}

Fuente: elaboración propia.

En cuanto a las puntuaciones de los participantes en los diferentes instrumentos de medida, observamos que obtuvieron una puntuación media en la percepción del dolor 
de $7.91(\mathrm{DT}=2.36)$, lo que indicaría un dolor severo. Con respecto a la calidad de vida los dos grupos, puntúan muy bajo en ambas áreas, especialmente en calidad de vida física. La autoeficacia percibida sobre el rango de 0 a 100 arrojó unas puntuaciones entorno al punto medio, siendo las puntuaciones más bajas en la autoeficacia para el control del dolor. Finalmente, sobre las variables relacionadas con el sueño, podemos destacar la baja cantidad de horas de sueño, poco más de cinco, y elevadas puntuaciones en alteraciones del sueño (Tabla 3).

\section{TABLA 3}

Diferencias de medias entre las puntuaciones del test y postest de las puntuaciones obtenidas en los instrumentos de evaluación

\begin{tabular}{|c|c|c|c|c|c|c|c|c|}
\hline \multicolumn{3}{|c|}{ Pre } & \multicolumn{2}{|c|}{ Post } & & & & \\
\hline $\mathrm{n}=105$ & M & DT & M & DT & $t$ & gl & $\mathrm{p}$ & d \\
\hline Dolor & 7.91 & 2.36 & 6.79 & 2.18 & 3.56 & 104 & 0.031 & 0.42 \\
\hline $\mathrm{CV}$ fisica & 29.65 & 7.36 & 30.27 & 8.22 & -0.92 & 104 & 0.359 & - \\
\hline $\mathrm{CV}$ mental & 31.96 & 10.96 & 31.54 & 11.01 & 2.99 & 104 & 0.004 & 0.28 \\
\hline $\mathrm{AE}$ sintomas & 43.85 & 18.7 & 51.89 & 20.88 & -4.88 & 104 & 0 & 0.43 \\
\hline AE fisico & 45.44 & 24.4 & 54.33 & 24.79 & -4.86 & 104 & 0 & 0.43 \\
\hline $\mathrm{AE}$ control d & 30.91 & 21.94 & 41.62 & 24.57 & -4.51 & 104 & 0 & 0.40 \\
\hline Ansiedad & 11.61 & 4.78 & 10.37 & 4.95 & 3.03 & 104 & 0.003 & 0.28 \\
\hline Depresión & 10.73 & 4.79 & 8.93 & 4.95 & 5.26 & 104 & 0 & 0.46 \\
\hline Alteraciones & 61.97 & 26.37 & 55.63 & 27.4 & 3.07 & 104 & 0.003 & 0.29 \\
\hline Cantidad & 5.59 & 1.56 & 5.92 & 1.56 & -2.74 & 104 & 0.007 & 0.26 \\
\hline Ronquidos & 46.54 & 32.67 & 42.88 & 31.09 & 1.05 & 104 & 0.297 & - \\
\hline Despertar br & 40 & 27.87 & 39.23 & 30.01 & 0.27 & 104 & 0.785 & - \\
\hline Somnolencia & 32.98 & 27.34 & 42.21 & 31.13 & -3.25 & 104 & 0.002 & 0.30 \\
\hline Idoneidad & 50.06 & 24.14 & 44.49 & 24.23 & 2.66 & 104 & 0.009 & 0.25 \\
\hline Interferencia & 56.7 & 21.33 & 50.48 & 23.86 & 3.11 & 104 & 0.002 & 0.29 \\
\hline Interferencia & 57.52 & 20.51 & 51.69 & 23.07 & 3.23 & 104 & 0.002 & 0.30 \\
\hline
\end{tabular}

Con la finalidad de analizar los efectos del programa de intervención multicomponente, se evaluó el cambio en los instrumentos de medida en el pret y postest, en cuanto a depresión, ansiedad, calidad de vida, autoeficacia y alteración del sueño, cuyos resultados se muestran en la Tabla 3 y se representan de forma gráfica en el Figura 1. Concretamente, se aprecian mejoras significativas con tamaños de efecto medios en las variables evaluación del dolor, componente mental de calidad de vida, autoeficacia en control de síntomas, autoeficacia en funcionamiento físico, autoeficacia en control del dolor, ansiedad y depresión. Con respecto a las variables que miden dimensiones relacionadas con el sueño, encontramos diferencias estadísticamente significativas con tamaños del efecto medios-bajos en alteraciones del sueño, cantidad y somnolencia.

\section{Figura 1.}

Diferencias de medidas entre las puntuaciones del test y postest de las puntuaciones obtenidas en los instrumentos de evaluación

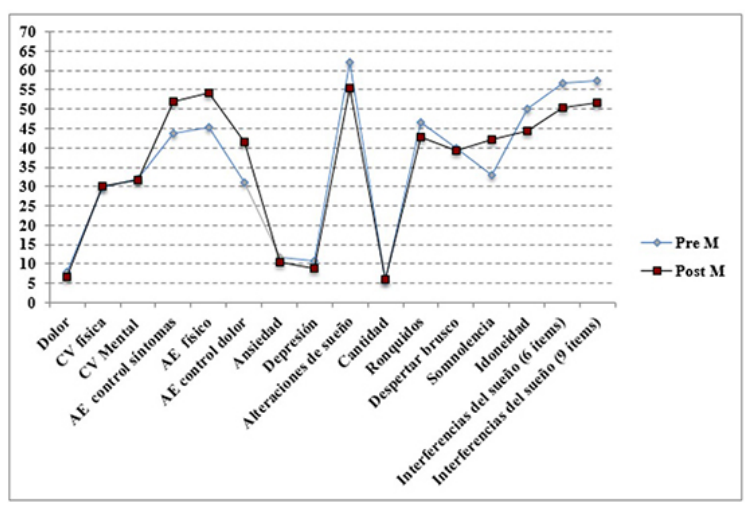

$$
\mathrm{CV}=\text { Calidad de vida } \mathrm{AE}=\text { Autoeficacia } .
$$

Fuente: elaboración propia.

\section{Discusión}

En este estudio se analizó la eficacia de un programa de intervención grupal multicomponente en una muestra de pacientes con dolor crónico.

El programa de intervención incluyó procedimientos de relajación y técnicas cognitivo-conductuales para controlar la intensidad con la que los participantes percibían el dolor. Así, se obtuvieron diferencias significativas entre los niveles de dolor previos a la aplicación del programa y los medidos al finalizar. Por tanto, podemos afirmar que el programa ayudó a reducir la percepción del dolor en los participantes. Son numerosos los estudios que defienden una intervención multicomponente para afrontar y reducir la percepción del dolor (Boschen et al., 2016; Eccleston et al., 2016; Inoue et al., 2014; McCracken, 2012; Moix \& Casado, 2011; Moix $\&$ Kovacs, 2009). Autores como Gombliewsky et al. (2010) explican que el tamaño del efecto que produce el tratamiento psicológico cognitivoconductual es igual de eficaz que el tratamiento farmacológico, con la diferencia de que las terapias psicológicas pueden mantener a un paciente estable hasta dos años. 
Por otro lado, al evaluar las variables psicológicas, se obtuvieron diferencias en los niveles de ansiedad, depresión, calidad de vida física, autoeficacia en todas sus dimensiones (control de síntomas, funcionamiento físico y control del dolor) (véase Tabla 3 y Figura 1). Esto se corresponde con lo reflejado en otros estudios que encuentran resultados similares los nuestro (Denison, Åsenlöf, \& Lindberg, 2004; Flink, Smeets, Bergbom, \& Peters, 2015; Inoue et al., 2014; Oliver, Fisher, \& Childs, 2016).

En cuanto a los niveles de atributos del sueño, observamos que los resultados sugieren mejorías en aspectos como cantidad de sueño y alteraciones del sueño (véase Tabla 3). Algunos estudios defienden que los trastornos del sueño presentan una relación directa y recíproca con la intensidad de dolor que sufre el paciente: el dolor produce mala calidad del sueño y este último, a su vez, conlleva una reducción de las horas de sueño (Lautenbacher, Kundermann, \& Krieg, 2006). En contraposición, detectamos un aumento de la somnolencia, que puede deberse a que percibirse como menos ansioso aumenta la sensación de somnolencia.

Una de las principales limitaciones de este estudio es la dificultad para realizar un estudio con un diseño experimental, ya que dejaría a pacientes con dolor crónico fuera del programa de intervención. Esto presentaría problemas éticos, pues ya existe evidencia de los beneficios que tienen los programas de intervención grupal en estos pacientes. Además, derivado de este estudio, surge la reflexión de si, ante la ausencia de profesionales específicamente formados en el tratamiento del dolor, la aplicación de programas como el que presentamos por parte de profesionales en formación con un mínimo de entrenamiento (en nuestro caso, estudiantes en prácticas del Máster en Psicología General Sanitaria y psicólogos internos residentes, supervisados por personal de la Unidad de Psicología) consigue resultados satisfactorios.

En futuras investigaciones se intentará ampliar el seguimiento de los pacientes mediante un estudio longitudinal para valorar la eficacia del programa en diferentes momentos temporales y las variaciones en las variables psicológicas y los niveles de dolor.

Para finalizar, podemos concluir que el programa de intervención grupal multicomponente es eficaz para disminuir la percepción del dolor, aumenta la calidad de vida en su componente físico, mejora la autoeficacia en todas sus dimensiones (control de síntomas, funcionamiento físico y control del dolor) y la cantidad y alteraciones del sueño.

\section{Referencias}

Alonso, J., Regidor, E., Barrio, G., Prieto, L., Rodríguez, C., \& de la Fuente, L. (1998). Valores poblacionales de referencia de la versión española del Cuestionario de Salud SF-36. Medicina Clínica, 111, 410-416.

Asociación Médica Mundial. (2013). Declaración de Helsinki de la AMM. Principios éticos para las investigaciones médicas en seres humanos. Recuperado de http:/www.wma.net/es/30publications/ 10policies/b3/

Bettina, S. A., Alpers, G. W., Friedel, E., Kosmützky, G., Geier, A., \& Pauli, P. (2008). Affective pain modulation in fibromyalgia, somatoform pain disorder, back pain and health control. European Journal of Pain, 12(3), 329-338. http://dx.doi.org/10.1016/ j.ejpain.2007.06.007

Boschen, K. A., Robinson, E., Campbell, K. A., Muir, S., Oey, E., Janes, K., ... Katz, J. (2016). Results from 10 years of a CBT Pain Self-Management Outpatient Program for Complex Chronic Conditions. Pain Research and Management, 2016, 1-10. http://dx.doi.org/10.1155/2016/4678083

Breivik, H., Collett, B., Ventafridda, V., Cohen, D., \& Gallacher, D. (2006). Survey of chronic pain in Europe: Prevalence, impact on daily life, and treatment. European Journal of Pain, 10(4), 287-333. http:// dx.doi.org/10.1016/j.ejpain.2005.06.009

Cabrera, V., Martín-Aragón, M., Terol, M. C., Núñez, R., \& Pastor, M. A. (2015). La Escala de Ansiedad 
y Depresión Hospitalaria (HAD) en fibromialgia: análisis de sensibilidad y especificidad. Terapia Psicológica, 33(3), 181-193. http://dx.doi.org/10.4067/ S0718-48082015000300003

Cohen, J. (1988). Statistical power analysis for the behavioral science (2. ${ }^{\circ}$ ed.). Hillsdale, NJ: Lawrence Erlbaum Associates.

Denison, E., Åsenlöf, P., \& Lindberg, P. (2004). Self-efficacy, fear avoidance, and pain intensity as predictors of disability in subacute and chronic musculoskeletal pain patients in primary health care. Pain, 111, 245-52. http://dx.doi.org/10.1016/ j.pain.2004.07.001

Eccleston, C., Hearn, L., \& Williams, A. C. (2016). Psychological therapies for the management of chronic neuropathic pain in adults. Cochrane Database Systematic Reviews, 11. http:// dx.doi.org/10.1002/14651858.CD011259.pub2

Flink, I. K., Smeets, E., Bergbom, S., \& Peters, M. L. (2015). Happy despite pain: Pilot study of a positive psychology intervention for patients with chronic pain. Scandinavian Journal of Pain, 7, 71-79. http:// dx.doi.org/10.1016/j.sppain.2015.01.005

Glombiewsky, J. A., Sawyer, A. T., Gutermann, J., Koenig, K., Rief, W., \& Hofmann, S. G. (2010). Psychological treatments for fibromyalgia: A meta-analysis. Pain, 151, 280-288. http://dx.doi.org/10.1016/ j.pain.2010.06.011

Hays, R. D., Martin, S. A., Sesti, A. M., \& Spritzer, K. L. (2005). Psychometric properties of the medical outcomes study sleep measure. Sleep Medicine, 6(1), 41-44.

Inoue, M., Inoue, S., Ikemoto, T., Arai, Y. C., Nakata, M., Miyazaki, A., ... Ushida, T. (2014). The efficacy of a multidisciplinary group program for patients with refractory chronic pain. Pain Research and Management, 19(6), 302-308.

Lautenbacher, S., Kundermann, B., \& Krieg, J. C. (2006). Sleep deprivation and pain perception. Sleep Medicine Reviews, 10, 357-369. http://dx.doi.org/10.1016/ j.smrv.2005.08.001
Ley Orgánica 15/1999 de 13 de diciembre, de Protección de Datos de Carácter Personal. Boletín Oficial del Estado, n. ${ }^{-}$298, de 14 de diciembre de 1999.

Martín-Aragón, M., Pastor, M. A., RodríguezMarín, J., March, M. J., Lledó, A., López-Roig, S., \& Terol, M. C. (1998). Percepción de autoeficacia en dolor crónico: adaptación y validación de la Chronic Pain Self-Efficacy Scale. Revista de Psicología de la Salud, 11 (1/2), 53-75.

McCracken, L. (2012). Contextual cognitive behavioral therapy for chronic pain (including back pain). En M. I. Hasenbring, A. C. Rusu, \& D. C. Turk (Eds), From acute to chronic back pain: Risk factors, mechanisms, and clinical implications (pp. 547-563). Oxford: Oxford University Press.

Moix, J., \& Casado, M. I. (2011). Terapias psicológicas para el tratamiento del dolor crónico. Clínica y Salud, 22 (1), 41-50.

Moix, J., \& Kovacs, F. M. (2009). Manual del dolor. Tratamiento cognitivo conductual del dolor crónico. Barcelona: Paidós.

Oliver, S., Fisher, K., \& Childs, S. (2016). What psychological and physical changes predict patients' attainment of personally meaningful goals six months following a CBT based pain management intervention? Disability $\mathcal{E}$ Rehabilitation, 21, 1-7. http:// dx.doi.org/10.1080/09638288.2016.1226415

Palanca-Sánchez, I., Puig-Riera, M. M., Elola-Somoza, J., Bernal-Sobrino, J. L., Paniagua-Caparrós, J. L., \& Grupo de Expertos. (2011). Unidad de tratamiento de dolor: estándares y recomendaciones. Madrid: Ministerio de Sanidad, Política Social e Igualdad. Recuperado de http://portal.sedolor.es/con tenidos/100/adjuntos/gnpg34cc.pdf

Rodríguez-Marín, J., van-der Hofstadt, C. J., \& Couceiro, S. (2013). Intervención grupal en dolor crónico. Manual del terapeuta. Madrid: Grupo 5.

Sauer, S. E., Burris, J. L., \& Carlson, C. R. (2010). New directions in the management of chronic pain: Selfregulation theory as a model for integrative 
clinical psychology practice. Clinical Psychology Review, 30(6), 805-14. http:// dx.doi.org/10.1016/j.cpr.2010.06.008.

Soares-Weiser, K., Sola, I., Aromataris, E., Tornero, J., Margarit, C., \& Kleijnen, J. (2010). Epidemiology of chronic nonmalignant pain in Spain. Escrick: Kleijnen Systematic Reviews.

Ware, J. E., Kosinski, M., Turner-Bowker, D. M., \& Gandek, B. (2002). How to Score Version 2 of the SF-12 Health Survey (With a Supplement Documenting Version 1). Lincoln: QualityMetric Incorporated.

Zigmond, A. S. \& Snaith, R. P. (1983). The Hospital Anxiety and Depression Scale. Acta Psychiatrica Scandinavica, 67, 361-370.

\section{Notas}

* Artículo de investigación. 